PUPT-1499

hep-th/9409062

12th September 1994

\title{
Heterotic Coset Models
}

\author{
Clifford V. Johnson ${ }^{\dagger}$ \\ Joseph Henry Laboratories \\ Jadwin Hall \\ Princeton University \\ Princeton NJ 08544 \\ $U S A$
}

\begin{abstract}
A description is given of how to construct $(0,2)$ supersymmetric conformal field theories as coset models. These models may be used as non-trivial backgrounds for Heterotic String Theory. They are realised as a combination of an anomalously gauged Wess-Zumino-Witten model, right-moving supersymmetric fermions, and left-moving current algebra fermions. Requiring the sum of the gauge anomalies from the bosonic and fermionic sectors to cancel yields the final model. Applications discussed include exact models of extremal four-dimensional charged black holes and Taub-NUT solutions of string theory. These coset models may also be used to construct important families of $(0,2)$ supersymmetric Heterotic String compactifications. The Kazama-Suzuki models are the left-right symmetric special case of these models.
\end{abstract}

(To appear in Mod. Phys. Lett. 1995)

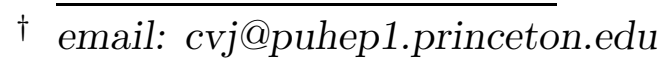




\section{Introduction and Motivation}

The aim here is to show how to construct non-trivial conformal field theories with $(0,2)$ supersymmetry. The motivation is clear: It is well known that in order to obtain the desired $N=1$ spacetime supersymmetry in heterotic string theory, the minimum requirement is world sheet $N=2$ supersymmetry. There are many well established results for the highly studied $(2,2)$ conformal field theories and many of the fascinating facts about their moduli spaces (e.g. Mirror Symmetry) have been uncovered to date. However, these models are in a sense over-specialised examples of the generic $(0,2)$ supersymmetric conformal field theories which heterotic string theory demands. In this sense, the task of studying the moduli space of heterotic string vacua has only just begun. A search for many $(0,2)$ models and understanding of their moduli has to begin in earnest. This letter will describe the construction of isolated points in this moduli space. We first describe the general case and then end with some examples and a brief discussion.

\section{2. $(2,2)$ cosets: Kazama-Suzuki Models}

Coset models were first invented by Bardakci and Halpern[1] and later generalised by Goddard, Kent and Olive[2] as algebraic realisations of new conformal systems, ' $G / H$ ' based upon affine Lie algebras (a special case of Kac-Moody algebras[3][4]) for a group $G$ and a subgroup $H$. The $N=1$ supersymmetric extension was worked out soon after and is based upon analogous constructions using affine Lie superalgebras[5]. (For a review see ref.[6].)

When the space $G / H$ is Kähler, it was shown by Kazama and Suzuki[7] using an algebraic construction that $N=1$ is promoted to an $N=2$ supersymmetry. This realises a large family of $(2,2)$ models, of which the $N=2$ minimal models (used for example by Gepner in his construction of non-trivial heterotic string vacua[8]) are the simplest case (they are realised as $S U(2) / U(1)$ ). The most straightforward examples are the 'hermitian symmetric spaces'.

For many reasons it is advantageous to have a Lagrangian definition of a conformal field theory which realises the algebraic structures as a field theory. It is very often 
a powerful supplement to the algebraic description. The gauged Wess-ZuminoWitten model is the appropriate device to use.

\section{3. $(2,2)$ Cosets as Gauged Wess-Zumino-Witten Models}

An action for a conformal field theory with all of the algebraic structures of the Kazama-Suzuki models is:

$$
\begin{aligned}
I^{(2,2)} & =I_{W Z W}(g)+I(g, A)+I_{F}\left(\Psi_{L}, \Psi_{R}, A\right)= \\
& -\frac{k}{4 \pi} \int_{\Sigma} d^{2} z \operatorname{Tr}\left[g^{-1} \partial_{z} g \cdot g^{-1} \partial_{\bar{z}} g\right] \\
& -\frac{i}{12 \pi} \int_{B} d^{3} \sigma \epsilon^{i j k} \operatorname{Tr}\left[g^{-1} \partial_{i} g \cdot g^{-1} \partial_{j} g \cdot g^{-1} \partial_{k} g\right] \\
& +\frac{k}{2 \pi} \int_{\Sigma} d^{2} z \operatorname{Tr}\left[A_{z} g^{-1} \partial_{z} g-A_{\bar{z}} \partial_{\bar{z}} g g^{-1}+A_{\bar{z}} g^{-1} A_{z} g-A_{z} A_{\bar{z}}\right] \\
& +\frac{i}{4 \pi} \int_{\Sigma} d^{2} z \operatorname{Tr}\left[\Psi_{+} \mathcal{D}_{\bar{z}} \Psi_{+}+\Psi_{-} \mathcal{D}_{z} \Psi_{-}\right]
\end{aligned}
$$

where the two dimensional surface $\Sigma=\partial B$ has complex coordinates $(z, \bar{z})$ and

$$
\begin{aligned}
g & \in G ; A^{a} \in \operatorname{Lie} H ; \\
\Psi_{ \pm} & \in \operatorname{Lie} G-\operatorname{Lie} H, \quad \mathcal{D}_{a} \equiv \partial_{a}+\left[A_{a}, \quad\right]
\end{aligned}
$$

and we have gauged the group invariance

$$
\begin{aligned}
g & \rightarrow h g h^{-1} \\
\Psi_{ \pm} & \rightarrow h \Psi_{ \pm} h^{-1} \\
A & \rightarrow h d h^{-1}+h A h^{-1}
\end{aligned}
$$

where $h(z, \bar{z}) \in H$.

This action has an $N=1$ supersymmetry:

$$
\begin{aligned}
& \delta g=i \epsilon_{-} g \Psi_{+}+i \epsilon_{+} \Psi_{-} g \\
& \delta \Psi_{+}=\epsilon_{-}\left(1-\Pi_{0}\right) \cdot\left(g^{-1} \mathcal{D}_{z} g-i \Psi_{+} \Psi_{+}\right) \\
& \delta \Psi_{-}=\epsilon_{+}\left(1-\Pi_{0}\right) \cdot\left(\mathcal{D}_{\bar{z}} g g^{-1}+i \Psi_{-} \Psi_{-}\right)
\end{aligned}
$$

where $\Pi_{0}$ is the orthogonal projection of Lie $G$ onto Lie $H$. 
Now, just as in the algebraic construction of Kazama and Suzuki, an $N=2$ supersymmetry arises from this $N=1$ when the space $G / H$ is Kähler. We will not dwell on this further here, save to note that this action was first studied in this context by Witten[9] and Nakatsu[10]. Witten used this action (after twisting) to do explicit calculations in certain topological field theories. The explicit $N=2$ transformations are written down in ref.[11] for example and there Henningson uses the models to study important properties of the Kazama-Suzuki models which are more easily accessible via field theoretic methods. This includes an investigation of mirror symmetry for the Kazama-Suzuki models and a calculation of the elliptic genus for the $N=2$ minimal models.

Note by the way that the bosonic and fermionic sectors in (3.1) are separately consistent models. In particular, the bosonic sector of the gauged Wess-ZuminoWitten (WZW) model is of course a consistent model realising the bosonic cosets[12] and the action for the chiral fermions, when written in this 'coset' basis, is just a simple minimal coupling to the gauge fields[13]. The chiral anomalies which potentially arise from this coupling exactly cancel due to the identical nature of the left and right fermion couplings. The anomalies contribute with opposite sign (due to opposite chirality) but equal magnitude.

\section{4. (0,2) Cosets: Potential Problems and a Solution}

(1) To get a $(0,2)$ conformal field theory, we need to remove the left $N=2$. Simply deleting or changing the couplings of the left moving fermions to the gauge fields would certainly do this for us, without spoiling the right-moving $N=2$. The only problem is that this procedure is bound to produce anomalies. The rightmovers' chiral anomaly will either have nothing to cancel against (if we deleted the left-movers), or will not completely cancel (if we changed the couplings of the left-movers to spoil the third symmetry in (3.2)).

(2) For many other reasons (as will be illustrated later), it would also be nice to gauge other symmetries of the WZW model. To get a consistent model, one has to gauge a restricted class of embeddings of subgroups of the full $G_{L} \times G_{R}$ symmetry 
which exists for the basic WZW. These are called 'anomaly-free' subgroups, mainly because one of the first uses of this type of model (in higher dimensional gauge theories) was to study the structure of anomalies[14] by deliberately studying anomalous subgroups, and then letting the Wess-Zumino term produce classically the familiar quantum gauge anomalies. Since Witten's paper on the use of the WessZumino term to define a conformally invariant sigma model in two dimensions - the Wess-Zumino-Witten model-most of the efforts involving them in $2 \mathrm{D}$, including their gauged versions, have made sure that there are no anomalies. This is simply because the model would not correctly reproduce the coset algebra - it would not be conformally invariant, in general.

Given the language just used to describe the problems we would like to solve, it is clear that a solution presents itself in the form of cancelling the anomalies against one another. If we arrange things correctly, this will work ${ }^{1}$. The next section describes just how to do this.

\section{Anomalies}

There are anomalies arising from three sectors now. The classical anomaly from the WZW and the chiral anomalies at one-loop from each chirality of fermion. We will discuss each in turn.

\section{The WZW anomalies.}

In general gauging the following symmetry of the WZW model

$$
\begin{aligned}
& \quad g \rightarrow h_{L} g h_{R}^{-1} \\
& \text { for }\left(h_{L}, h_{R}\right) \in\left(H_{L}, H_{R}\right) \subset\left(G_{L}, G_{R}\right)
\end{aligned}
$$

1 Indeed, the idea of achieving quantum consistency of chiral fermions by cancelling their anomalies against anomalies generated by Wess-Zumino terms goes back to Faddeev and Shatashvili, where it was studied both in four dimensions[15] and two dimensions[16]. The author is grateful to Samson Shatashvili for pointing this out. See also ref.[17]. 
is anomalous. This simply means that one cannot write down an extension of the WZW model which promotes this symmetry to a local invariance: There will always be terms which spoil gauge invariance. (This is because of the Wess-Zumino term; the 'metric' term may be simply minimally coupled.)

Knowing that we will get an anomaly, let us choose to write some gauge extension such that under gauge transformations the 'anomalous' piece does not depend upon the group element $g$. This results in the anomalous piece taking the form of the standard 2D chiral anomaly. The unique action is[18]:

$$
\begin{aligned}
I_{G W Z W}^{G_{k}}(g, A) & =-\frac{k}{4 \pi} \int_{\Sigma} d^{2} z \operatorname{Tr}\left[g^{-1} \mathcal{D}_{z} g \cdot g^{-1} \mathcal{D}_{\bar{z}} g\right] \\
& -\frac{i k}{12 \pi} \int_{B} d^{3} \sigma \epsilon^{i j k} \operatorname{Tr}\left[g^{-1} \partial_{i} g \cdot g^{-1} \partial_{j} g \cdot g^{-1} \partial_{k} g\right] \\
& -\frac{k}{4 \pi} \int_{\Sigma} A^{a} \wedge \operatorname{Tr}\left[t_{a, L} \cdot d g g^{-1}+t_{a, R} g^{-1} d g\right] \\
& -\frac{k}{8 \pi} \int_{\Sigma} A^{a} \wedge A^{b} \operatorname{Tr}\left[t_{a, R} g^{-1} t_{b, L} g-t_{b, R} g^{-1} t_{a, L} g\right] .
\end{aligned}
$$

Under the infinitesimal variation

$$
\begin{aligned}
g & \rightarrow g+\epsilon^{L} g-g \epsilon^{R} \\
A^{R(L)} & \rightarrow A^{R(L)}-d A^{R(L)}-\left[A^{R(L)}, \epsilon^{R(L)}\right]
\end{aligned}
$$

where

$$
\begin{aligned}
\epsilon^{R(L)} & \equiv \epsilon^{a} t_{a, R(L)} \\
A^{R(L)} & \equiv A^{a} t_{a, R(L)} \\
D g & \equiv d g+A^{L} g-g A^{R},
\end{aligned}
$$

the variation is

$$
\begin{aligned}
& \delta I(g, A)=\frac{k}{4 \pi} \operatorname{Tr}\left[t_{a, R} \cdot t_{b, R}-t_{a, L} \cdot t_{b, L}\right] \int_{\Sigma} d^{2} z \epsilon^{(a)} F_{z \bar{z}}^{(b)} \\
& \text { where } t_{a, L(R)} \in \operatorname{Lie} H_{L(R)} .
\end{aligned}
$$

Notice in particular that for the popular diagonal gaugings of WZW models this variation is zero and the action reduces to the familiar one. 


\section{The right movers}

As mentioned before, it is sufficient to minimally couple the coset fermions to the gauge fields:

$$
\begin{aligned}
& I_{F}^{R}\left(\Psi_{R}, A\right)=\frac{k}{4 \pi} \int_{\Sigma} i \operatorname{Tr}\left[\Psi_{R} \mathcal{D}_{\bar{z}} \Psi_{R}\right] \\
& \text { where } \mathcal{D}_{\bar{z}} \Psi_{R}=\partial_{\bar{z}} \Psi_{R}+\sum_{a} A_{\bar{z}}^{a}\left[t_{a, R}, \Psi_{R}\right], \Psi_{R} \in \operatorname{Lie} G-\operatorname{Lie} H
\end{aligned}
$$

There are $D=\operatorname{dim} G-\operatorname{dim} H$ fermions $\psi_{R}^{i}$ in $\Psi_{R}$, all coupled with charges derived from the generators $t_{a, R}$. The chiral anomalies appear at one loop and $\mathrm{are}^{2}$ :

$$
\frac{1}{4 \pi} \operatorname{Tr}\left[t_{a, R} \cdot t_{b, R}\right] \int_{\Sigma} d^{2} z \epsilon^{(a)} F_{z \bar{z}}^{(b)}
$$

(Note here the absence of $k$, which plays the role of $1 / \hbar$. This really is a one loop effect.)

\section{The left movers}

Let us couple into the model some left movers. Let us add $D=\operatorname{dim} G-\operatorname{dim} H$ of them (a good choice, as we will see later) with arbitrary couplings. To be precise, arrange them into a fundamental vector $\Lambda_{L}=\left\{\lambda_{L}^{i}\right\}$ of the group $S O(D)_{L}$ which acts on them as a global symmetry, and minimally couple them to the $H_{L}$ subgroup with generators $Q_{a, L}$ in this fundamental representation:

$$
I_{F}^{L}\left(\lambda_{L}^{i}, A\right)=\frac{k}{4 \pi} \int_{\Sigma} i \Lambda_{L}^{T}\left(\partial_{z}+\sum_{a} A_{z}^{a} Q_{a, L}\right) \Lambda_{L} .
$$

Their chiral anomalies appear at one loop and are:

$$
-\frac{1}{4 \pi} \widetilde{\operatorname{Tr}}\left[Q_{a, L} \cdot Q_{b, L}\right] \int_{\Sigma} d^{2} z \epsilon^{(a)} F_{z \bar{z}}^{(b)}
$$

2 Here and for the remainder of the letter, it is implicit that a consistent regularisation scheme has been chosen for calculation of the fermion anomalies, and such that the normalisation of the anomalies is chosen to be of this simple form. 
(Here $\widetilde{T r}$ is the trace in the fundamental representation of $S O(D)$. Note again the absence of $k$. Also note the minus sign relative to (5.4), due to the opposite chirality.)

So if we add together the three actions (5.1),(5.3) and (5.5), we get a gauge invariant model if we ensure that all of the anomalies (classical and quantum) cancel:

$$
k \operatorname{Tr}\left[t_{a, R} \cdot t_{b, R}-t_{a, L} \cdot t_{b, L}\right]+\operatorname{Tr}\left[t_{a, R} \cdot t_{b, R}\right]-\widetilde{\operatorname{Tr}}\left[Q_{a} \cdot Q_{b}\right]=0
$$

Our model has $(0,2)$ supersymmetry as advertised (because we have not touched the right-moving sector), and is conformally invariant.

Well, our model is gauge invariant when we take into account the one-loop effects, but we still have not written a classically gauge invariant action. This means that we cannot truly carry out procedures like path-integral quantisation, etc. We have not quite achieved our goal of a Lagrangian realisation of a $(0,2)$ conformal field theory.

The answer is to bosonize the fermions. The bosonic action equivalent to $I_{R}^{F}+I_{L}^{F}$ is classically anomalous. It is a theory of $D / 2$ real bosons with the same anomalies as above.

\section{Bosonisation}

In the specific examples to be mentioned later, the bosonisation was worked out 'by hand' and was for abelian cases. After a little thought, however, it is clear once one realises that a classically anomalous bosonic theory equivalent to an anomalous fermionic theory is to be found, it might be that the bosonic theory is something like another anomalously gauged WZW. This can be seen as follows.

Note that before gauging there are $D$ free fermions on the left and right. They therefore carry a global $S O(D)_{L} \times S O(D)_{R}$ symmetry. Witten showed in ref.[19] that this system of free fermions is equivalent to a Wess-Zumino-Witten model based on $S O(D)$ at level 1. Considering what we saw about WZW anomalies in section 5 it is clear that the classically anomalous bosonic theory equivalent to 
the fermionic theory is just this $S O(D)$ WZW gauged anomalously with different embeddings of $H$ in $S O(D)$ on the left and on the right:

$$
\begin{aligned}
\tilde{g} & \rightarrow \tilde{h}_{L} \tilde{g} \tilde{h}_{R}^{-1} \\
\text { for } \tilde{g} & \in S O(D) \text { and } \\
\left(\tilde{h}_{L}, \tilde{h}_{R}\right) & \in\left(H_{L}, H_{R}\right) \subset\left(S O(D)_{L}, S O(D)_{R}\right)
\end{aligned}
$$

Let the $\left(H_{L}, H_{R}\right)$ be generated by $\left(Q_{a, L}, Q_{a, R}\right)$. Choose the $Q_{a, R}$ such that when acting on the $\psi_{R}^{i}$ 's in the fundamental representation of $S O(D)$ they are equivalent to the $t_{a, R}$ acting on the $\psi_{R}^{i}$ in the coset fermion $\Psi_{R} \in \operatorname{Lie} G-\operatorname{Lie} H$. This will ensure that the right moving fermions are correctly coupled and preserve the (now hidden) $N=2$ on the right.

Then the bosonic action equivalent to the interacting fermions is just an action of the form (5.1) (with level 1), which yields the classical anomalies:

$$
\frac{1}{4 \pi} \widetilde{\operatorname{Tr}}\left[Q_{a, R} \cdot Q_{b, R}-Q_{a, L} \cdot Q_{b, L}\right] \int_{\Sigma} d^{2} z \epsilon^{(b)} F_{z \bar{z}}^{(a)} .
$$

So cancelling this against the anomaly of the $G / H$ bosonic model (and recalling from the above paragraph that $\left.\widetilde{\operatorname{Tr}}\left[Q_{a, R} \cdot Q_{b, R}\right]=\operatorname{Tr}\left[t_{a, R} \cdot t_{b, R}\right]\right)$, we recover (5.7) as the condition for a consistent model.

\section{7. $(0,2)$ Cosets as Gauged Wess-Zumino-Witten Models}

So finally we can write a classically gauge invariant analogue of (3.1) which realises a $(0,2)$ conformal field theory as a gauge invariant action written as the sum of two gauged Wess-Zumino-Witten models which are separately anomalous:

$$
I^{(0,2)}=I_{G W Z W}^{G_{k}}(g, A)+I_{G W Z W}^{S O(D)_{1}}(\tilde{g}, A),
$$

where $D=\operatorname{dim} G-\operatorname{dim} H$.

The heterotic coset is realised as: $\left[G_{k} \times S O(D)_{1}\right] / H$ with the gauged symmetry:

$$
\begin{gathered}
g \rightarrow h_{L} g h_{R}^{-1} \\
\tilde{g} \rightarrow \tilde{h}_{L} \tilde{g} \tilde{h}_{R}^{-1} \\
\text { subject to } k \operatorname{Tr}\left[t_{a, R} \cdot t_{b, R}-t_{a, L} \cdot t_{b, L}\right]+\operatorname{Tr}\left[t_{a, R} \cdot t_{b, R}\right]-\widetilde{\operatorname{Tr}}\left[Q_{a, L} \cdot Q_{b, L}\right]=0 .
\end{gathered}
$$


Note that $h_{R}$ and $\tilde{h}_{R}$ are chosen so as to recover right-moving supersymmetry in the fermion picture.

Note that in (7.1) the gauge extensions to each WZW (written using (5.1)) are generally not gauge invariant, but together they are because of the anomaly equation

above. In the special case of $h_{L}=h_{R}$ and $\tilde{h}_{L}=\tilde{h}_{R}$, they are each separately gauge invariant extensions, the anomaly equation is trivially satisfied, and we recover the $(2,2)$ case, the Kazama-Suzuki models. In this sense, the $(2,2)$ models can now be seen as a special case of a more general class of $(0,2)$ models.

\section{Some examples.}

These ideas were originally used by the author to study some particular cases[20]. The prototype model for this construction is the 'monopole theory' of Giddings, Polchinski and Strominger[21] (GPS). It is a conformal field theory of a heterotic string in a Dirac monopole background of charge $Q$ on a two-sphere of radius $Q$. GPS described it as an asymmetric orbifold of $S U(2)$. In ref.[20], when described as a heterotic coset, it is based upon an $S U(2)$ WZW with the $U(1)$ subgroup of the right $S U(2)$ gauged. Adding supersymmetric right movers and left movers of charge $Q$ gives an anomaly equation $k=2\left(Q^{2}-1\right)$. Bosonising the fermions it is possible to correctly determine the quadratic terms in the gauge fields which turns out to only depend upon $Q$. After integrating out the gauge fields (valid for large $Q$ ), and correctly re-fermionising the action, the heterotic sigma model describing the above system is recovered. This is described in detail in ref.[20]. As pointed out by GPS, the tensor product of this model with a supersymmetric $S L(2, \mathbb{R}) / U(1)$ $2 \mathrm{D}$ black hole coset[22] yields a $4 \mathrm{D}$ solution which is the extremal limit of the magnetically charged dilaton black hole of Gibbons, Maeda and Garfinkle, Horowitz and Strominger[23].

Notice that in the construction just described for the monopole theory, one cannot have a charge $Q=0$ solution, as then the anomaly equation would not be satisfied. After a little thought, it is clear that there is a quick way out of this problem: simply gauge $g \rightarrow h g$ instead and keep everything else the same. Then the sign of the WZW anomaly changes and the condition $k=2\left(1-Q^{2}\right)$ should now be satisfied. Now it 
is possible to get a $Q=0$ solution. (In constructing their neutral solution in their paper, Giddings, Polchinski and Strominger arrive at this simple modification in an equivalent way. This is indeed the same solution). Now naively, the interpretation of the model would be as a heterotic string on a neutral two-sphere background. However, it is easy to see that this is wrong. The problem of incorrectly identifying the two-sphere as the background manifold for small $Q$ has its roots in the fact that the final form of the metric for the model is obtained by integrating out the constraining 2D gauge fields, a process which is well defined only for large $Q$. which is equivalent to small $\alpha^{\prime}$, or large $k$. Here, the neutral solution has $k=2$, and no sensible metric interpretation may be made of the target space via perturbation theory, as all length scales (in units of $\alpha^{\prime}$ ) contribute equally to the $\beta$-function equations.

In the light of the work of GPS, the most obvious application of this construction was to find more general 4D solutions which were dyons (i.e. with both magnetic and electric charge). Applying this construction to general gaugings of $S L(2, \mathbb{R})$ was carried out in ref.[20], generalising Witten's conformal field theory of a 2D black hole[22] by yielding the conformal field theory of the known $2 \mathrm{D}$ charged black hole heterotic string solutions of McGuigan, Nappi and Yost[24]. Then 4D dyonic solutions were defined by tensor product with the GPS theory. At about the same time, Lowe and Strominger wrote a paper[25] about $4 \mathrm{D}$ dyons which were defined by tensoring the GPS theory with an asymmetric orbifold of $S L(2, \mathbb{R})$. This asymmetric orbifold may be realised an $S L(2, \mathbb{R})$ heterotic coset.

Instead of tensor products of these $2 \mathrm{D}$ theories, it is possible to obtain $4 \mathrm{D}$ dyon solutions which are not tensor products, by gauging (for example) a $U(1) \times U(1)$ subgroup of $S L(2, \mathbb{R}) \times S U(2)$ embedded non-trivially such that the action of the $U(1)$ 's was shared among the two parent groups. In a $4 \mathrm{D}$ dyon with a non-trivial mixing of the angular and radial coordinates was obtained in ref.[20]. This solution is the extremal limit of a dyonic, axionic analogue of the Taub-NUT solution of General Relativity, as confirmed by Myers and the author in ref.[26] and also by Kallosh, Kastor Ortin and Torma [27]. It would have been difficult to construct such a non-trivial solution as a conformal field theory without the use of the heterotic coset technique. 


\section{Future directions}

There are a huge number of avenues opened by allowing such freedom to gauge any subgroup of the WZW model's symmetries, obtaining consistency by adding heterotic fermions. One general point is that it allows one to consider leaving important WZW symmetries untouched, which in turn leaves certain spacetime symmetries intact. For example, in the simple GPS monopole model (and its neutral cousin) in the last section, leaving the $S U(2)_{L}\left(\right.$ or $\left.S U(2)_{R}\right)$ action untouched meant that a spacetime spherically symmetric system was obtained from an $S U(2)$ WZW. This type of freedom will certainly lead to many more interesting heterotic string backgrounds. The search for more 4D cosmological heterotic string backgrounds seems a promising area to apply this technique to.

It is worth noting here that the presence of the right moving supersymmetry is of course not neccessary for the consistency of these models. It is present in this paper only because the of the context of superstrings. Indeed, one may simply relax the requirement of the right $N=2$ and give the right moving fermions arbitrary couplings also. As long as the anomalies cancel, this will still produce consistent conformal field theories, with an asymmetric combination of left and right characters for the partition functions. The values of the fermion couplings which correspond to $(0,2),(2,0)$ and $(2,2)$ conformal field theories would then be regarded as special points in the moduli space of non-supersymmetric backgrounds. The $(0,2)$ monopole theory described in the previous section was first described by GPS as a such a special point in a family of non-supersymmetric models.

Of great interest is the problem of calculating the spectrum and partition function for these models. This will be of course a highly non-trivial combination of right $N=2$ characters and general $N=0$ characters. It is a hard problem to discover the heterotic modular invariant combinations algebraically of course (see e.g. ref.[28]), but there are promising signs that their Lagrangian description using a gauged WZW with fermions, as described here may provide some guidance. Work is in progress on this and related matters with Berglund, Kachru and Zaugg[29].

The problem of starting to map out the moduli space of $(0,2)$ models can be 
attacked successfully by studying the marginal perturbations of these models. This is of course much easier when there exists a Lagrangian description of the type constructed here. Such marginal perturbations would help to find the geometrical interpretation of the nieghbourhoods of these models, in the case of their use as string compactifications.

Marginal perturbations would also represent interesting geometrical freedom in some 4D solutions, where they correspond to such processes as widening the throat of some of the extremal solutions of the type mentioned in the last section, connecting onto the asymptotically flat 4D exterior solution[21].

There are of course many more questions which need to be answered about the moduli space of $(0,2)$ conformal field theories. Hopefully this construction may go some way to help to answer them.

\section{Acknowledgements}

I am grateful for the hospitality of the International Centre For Theoretical Physics at Trieste during my stay there in July 1994, where many of the details of this letter were worked out and presented as a talk in the Summer School and Workshop on High Energy Physics and Cosmology.

I would also like once again to thank Ed Witten for originally pointing out to me well over a year ago that the GPS monopole theory might be described by a construction of the type described in this talk.

This work was supported by an EPSRC (UK) NATO Fellowship. 


\section{References}

[1] K. Bardakci and M. B. Halpern, Phys. Rev. D3 (1971) 2493;

M. B. Halpern, Phys. Rev. D4 (1971) 2398.

[2] P. Goddard and D. Olive, Nucl. Phys. B257 (1985) 226;

P Goddard, A Kent and D Olive, Phys Lett B152 (1985) 88;

P Goddard, A Kent and D Olive, Commun Math Phys 103 (1986) 105.

[3] V. G. Kac, Funct. Anal. App. 1 (1967) 328;

R. V. Moody, Bull. Amer. Math. Soc. 73 (1967) 217.

[4] V. G. Kac, 'Infinite-dimensional Lie Algebras - An Introduction', Birkhäuser, Basel 1983, 2nd Edition Cambridge University Press, Cambridge 1985.

[5] V. G. Kac and T. Todorov, Commun. Math. Phys. 103 (1986) 105.

[6] P. Goddard and D. Olive, Int. Jour. Mod. Phys. A1 (1986) 303.

[7] Y. Kazama and H. Suzuki, Nucl. Phys. B321 (1989) 232;

Y. Kazama and H. Suzuki, Phys. Lett. B216 (1989) 112.

[8] D. Gepner, Nucl. Phys. B290 (1987) 10;

D. Gepner, Nucl. Phys. B296 (1988) 757.

[9] E. Witten, Nucl. Phys. B371 (1992) 191.

[10] S. Nakatsu, Prog. Theor. Phys. 87 (1992) 795.

[11] M. Henningson, Nucl. Phys. B413 (1994) 73;

M. Henningson, Institute for Advanced Study preprint IASSNS-HEP-94/13, hepth/9402122.

[12] See for example D. Karabali, Q.-H. Park, H. Schnitzer and Z. Yang, Phys. Lett. B216 (1989) 307.

[13] R. Rohm, Phys. Rev. D32 (1985) 2849.

[14] See for example the book 'Current Algebra and Anomalies', S. B. Treiman, E. Witten, R. Jackiw and B. Zumino, World Scientific, Singapore 1986.

[15] L. D. Faddeev, Phys. Lett. B145 (1984) 81.;

L. D. Faddeev and S. L. Shatashvili, Theor. Math. Phys. 60 (1985) 770; 
L. D. Faddeev and S. L. Shatashvili, Phys. Lett. B167 (1986) 225.

[16] S. L. Shatashvili, Theor. Math. Phys. 71 (1987) 366.

[17] I. G. Halliday, E. Rabinovici, A. Schwimmer and M. Chanowitz, Nucl. Phys. 268 (1986) 413.

[18] E. Witten, Commun. Math. Phys. 144 (1992) 191.

[19] E. Witten, Commun Math Phys 92 (1984) 455

[20] C. V. Johnson, Phys. Rev. D50 (1994) 4032, hep-th/9403192

[21] S. Giddings, J. Polchinski and A. Strominger, Phys. Rev. D48 (1993) 5784. hepth/9405083

[22] E. Witten, Phys. Rev. D44 (1991) 314.

[23] G. W. Gibbons and K. Maeda, Nucl. Phys. B298 (1988) 741;

D. Garfinkle, G. T. Horowitz and A. Strominger, Phys. Rev. D43 (1991) 3140, erratum Phys. Rev. D45 (1992) 3888.

[24] M. D. McGuigan, C. R. Nappi and S. A. Yost, Nucl. Phys. B375 (1992) 421. hep-th/9111038.

[25] D. A. Lowe and A. Strominger, Phys. Rev. Lett. 73 (1994) 1468, hep-th/9403186.

[26] C. V. Johnson and R. C. Myers, Phys. Rev, D50 (1994) 6512, hep-th/9406069.

[27] R. Kallosh, D. Kastor, T. Ortin and T. Torma, Phys. Rev, D50 (1994) 6374, hep-th/9406059.

[28] See for example T. Gannon, Nucl. Phys. B402 (1993) 729. hep-th/9209042.

[29] P. Berglund, C. V. Johnson, S. Kachru and P. Zaugg, 'Heterotic Coset Models II: The spectrum of $(0,2)$ conformal field theories', in preparation. 